\title{
Totally implantable venous access devices in children with cystic fibrosis: incidence and type of complications
}

\author{
J Deerojanawong, S M Sawyer, A M Fink, K B Stokes, C F Robertson
}

\begin{abstract}
Background-Totally implantable vascular access devices (TIVADs) are accepted as a safe and effective method of facilitating long term intravenous therapy. We report our experience of the use of these devices in children with cystic fibrosis with a particular focus on the incidence and type of complications.

Methods-The medical records of patients with cystic fibrosis who underwent placement of a TIVAD at the Royal Children's Hospital, Melbourne, Australia from January 1987 to October 1996 were reviewed. Venous ultrasonography with Doppler was performed in surviving patients with a TIVAD in situ from November 1996 to April 1997 to detect occult thrombotic complications.

Results-A total of 57 TIVADs were implanted in 44 children with a median functional duration of 700 days (range 27-3347 days). Twenty one children had devices inserted without complications. Forty eight complications (30 mechanical, 18 infectious) occurred in 36 devices in 23 children during a total functional duration of 53057 catheter days. Mechanical complications occurred in $53 \%$ of devices (one per 1712 catheter days). Symptomatic venous thrombosis occurred five times in four patients (9\%). Infectious complications occurred in 32\% (one per 2948 catheter days) while sepsis occurred in five devices (9\%). Doppler ultrasonography detected unsuspected thrombosis in two of 10 patients examined.
\end{abstract}

Conclusions-While TIVADs provided effective long term intravenous access, septic and thrombotic complications caused significant morbidity in this population. Careful patient selection, adherence to aseptic technique for access and blood sampling, and periodic ultrasonography with Doppler to detect early thrombosis may help reduce these risks. (Thorax 1998;53:285-289)

Royal Children's Melbourne, Victoria 3052, Australia

Correspondence to: Dr C Robertson.

Received 12 September 1997 Returned to authors 10 November 1997 Revised version received 3 December 1997 Accepted for publication 23 December 1997
Intravenous antibiotic therapy for exacerbations of respiratory infection in children with cystic fibrosis is an established and effective form of therapy. A safe and effective method of venous access is important in the care and treatment of these children, especially for those who require frequent courses of intravenous antibiotics. Totally implantable vascular access devices (TIVADs) have been successfully used in oncological patients requiring long term intravenous chemotherapy. These devices require minimal care, do not limit physical activity, and are relatively tamper-proof. The use of TIVADs in patients with cystic fibrosis was first described in $1986 .{ }^{1}$ Since then, reports show low complication rates for infection but a higher prevalence of thrombotic complications. $^{2-6}$ We report our experience with the use of TIVADs in children with cystic fibrosis over a nine year period, focusing on the incidence and type of complications.

\section{Methods}

We performed a retrospective review of all children with cystic fibrosis who had one or more TIVADs inserted at the Royal Children's Hospital, Melbourne, Australia between January 1987 and October 1996. The indications for insertion of these devices were frequency of hospitalisation, multiple peripheral venous lines during a single admission, and increasing difficulty with venous access. All TIVADs (Infus-a-Port, Strato/Infusaid Inc, Norwood, Massachusetts, USA) were inserted surgically under general anaesthesia. Catheters were inserted through facial or internal jugular veins and positioned under fluoroscopy so that the tip was in the superior vena cava. The porta was inserted subcutaneously on the anterior chest wall. These devices were used chiefly for antibiotic administration, although blood sampling was performed occasionally. Monthly flushing of the TIVAD with $5 \mathrm{ml}$ heparin saline solution $(100 \mathrm{U} / \mathrm{ml})$ took place during a routine outpatient clinic visit or by a visiting district nurse in the home. A Huber non-coring needle was used to penetrate the septum.

Medical records of all patients were reviewed. Demographic data, operative summaries, pulmonary function tests at the time of first insertion, duration of device, complications related to the device, and indication for removal of the device were sought. Data available on 30 April 1997 was analysed, providing a minimum follow up of six months. During the time of this study 38 children were managed at the Royal Children's Hospital and six young adult patients were managed at the Alfred Hospital. Grey scale and Doppler ultrasonography was performed in surviving patients with a TIVAD between November 1996 and April 1997 to detect silent thrombotic complications. Eight studies were performed at 
Table 1 Type of complications resulting from use of totally implantable vascular access devices $(n=48)$

\begin{tabular}{llcc}
\hline $\begin{array}{l}\text { Type of } \\
\text { complication }\end{array}$ & Nature of complication & $\begin{array}{l}\text { Number of } \\
\text { episodes }\end{array}$ & $\begin{array}{l}\text { Cause of } \\
\text { removal }\end{array}$ \\
\hline Mechanical & Catheter occlusion & 13 & 7 \\
& Symptomatic venous thrombosis & 5 & 5 \\
& Asymptomatic venous obstruction & 2 & - \\
& Catheter displacement & 4 & - \\
& Pain at port site & 2 & - \\
& Ruptured port & 1 & 1 \\
& Displaced port & 1 & - \\
& Discomfort & 1 & - \\
& Dermatitis & 1 & - \\
& Total & 30 & - \\
& & 13 & 2 \\
& Site infection & 5 & 3 \\
& $\quad+$ ve culture & 8 & 5 \\
& -ve culture & 5 & 2 \\
& Systemic infection & 3 & \\
& +ve culture from port & 18 &
\end{tabular}

the Royal Children's Hospital by one radiologist. The remaining two patients were studied at the Alfred Hospital.

\section{Results}

Forty seven patients with cystic fibrosis had one or more TIVAD placed over the nine year period. Three patients were excluded from the study because they were no longer in contact with either the paediatric or adult cystic fibrosis centre. Fifty seven devices were implanted in 44 patients with a median age at first insertion of 13 years (range 3-19 years). Thirty three children $(75 \%)$ had received one TIVAD, $10(23 \%)$ received two, and one child received four. There were no intraoperative complications.

Lung function tests were performed before first implantation in 40 of the 44 children. The median forced vital capacity (FVC) \% predicted value was $80 \%$ (range 35-107) and the median forced expiratory volume in one second $\left(\mathrm{FEV}_{1}\right) \%$ predicted value was $64 \%$ (range 31-111).

The duration of function of the devices ranged from 27 days to 3347 days (median 700 days). The cumulative total of catheter days was 53057 days. The median functional dura-

Table 2 Functional duration of catheters removed because of complications

\begin{tabular}{ll}
\hline Functional duration (days) & Reason for removal \\
\hline 27 & Infection \\
56 & Thrombosis \\
71 & Thrombosis \\
84 & Blocked \\
141 & Ruptured port \\
143 & Displaced \\
250 & Discomfort \\
311 & Infection \\
437 & Displaced \\
497 & Thrombosis \\
506 & Blocked \\
649 & Blocked \\
697 & Infection \\
822 & Blocked \\
888 & Infection \\
1054 & Fungal infection \\
1083 & Thrombosis \\
1122 & Displaced \\
1151 & Blocked \\
1185 & Blocked \\
1230 & Thrombosis \\
2039 & Blocked \\
2341 & Blocked \\
\hline
\end{tabular}

tion of the first device was 690 days (range 71-3347). Forty eight complications occurred during the study time. The details of the complications are summarised in table 1 .

\section{MECHANICAL COMPLICATIONS}

There were 30 mechanical complications. The median duration of catheter insertion before a mechanical complication was 592 days (range 56-3348). Eighteen of these catheters (60\%) were subsequently removed (table 2). The median duration of catheter insertion before removal for mechanical complication was 578 days (range 56-2341).

Catheter occlusion (unable to infuse or withdraw) was a common mechanical complication and occurred on 13 occasions. Heparin saline relieved the obstruction on five of these 13 occasions. A streptokinase infusion was used on four occasions after failed heparin saline flush. One was successfully cleared, but the remaining three devices remained occluded and were surgically removed. One "occluded" device was found to have a ruptured portal following surgical removal.

Symptomatic venous thrombosis developed in four children $(9 \%)$ on five occasions. All were diagnosed by Doppler ultrasonography and radiographic contrast studies. Three children developed thrombosis around their first device 65,1082 , and 1226 days, respectively, after the catheter was inserted. The other two children developed thrombosis around their second device 64 and 497 days after insertion. The latter patient had one previous episode of thrombosis in the first device, with the second device placed six months after removal of the first. All children were symptomatically relieved with streptokinase or local tissue plasminogen activator infusion and fully heparinised. However, all devices with thrombosis were eventually removed and all patients received anticoagulant therapy for a variable period. Two children presented with pain during injection and fullness of the neck. Both developed thrombosis in the right internal jugular vein 65 and 1226 days, respectively, after placement of their first TIVAD.

One patient had superior vena cava obstruction. This patient had her first TIVAD removed from the right internal jugular vein because of catheter blockage. The removal procedure was difficult and the catheter fractured. A replacement catheter was inserted at the same operation into the right internal jugular vein. Nine weeks later she presented with facial swelling. On ultrasound examination the catheter was found to be coursing along the left brachiocephalic vein with the tip in the left internal jugular vein and this was confirmed on review of the chest radiograph. An extensive thrombus was demonstrated in the left internal jugular vein, left subclavian vein, and brachiocephalic vein. Three fragments of the initial catheter, measuring $3 \mathrm{~cm}, 1 \mathrm{~cm}$, and $9 \mathrm{~mm}$, were also seen in the right internal jugular vein in the lower neck and superior mediastinum, lying against the posterior wall of the vein. A stenosis of the right internal jugular vein was noted at the level of the most central residual 

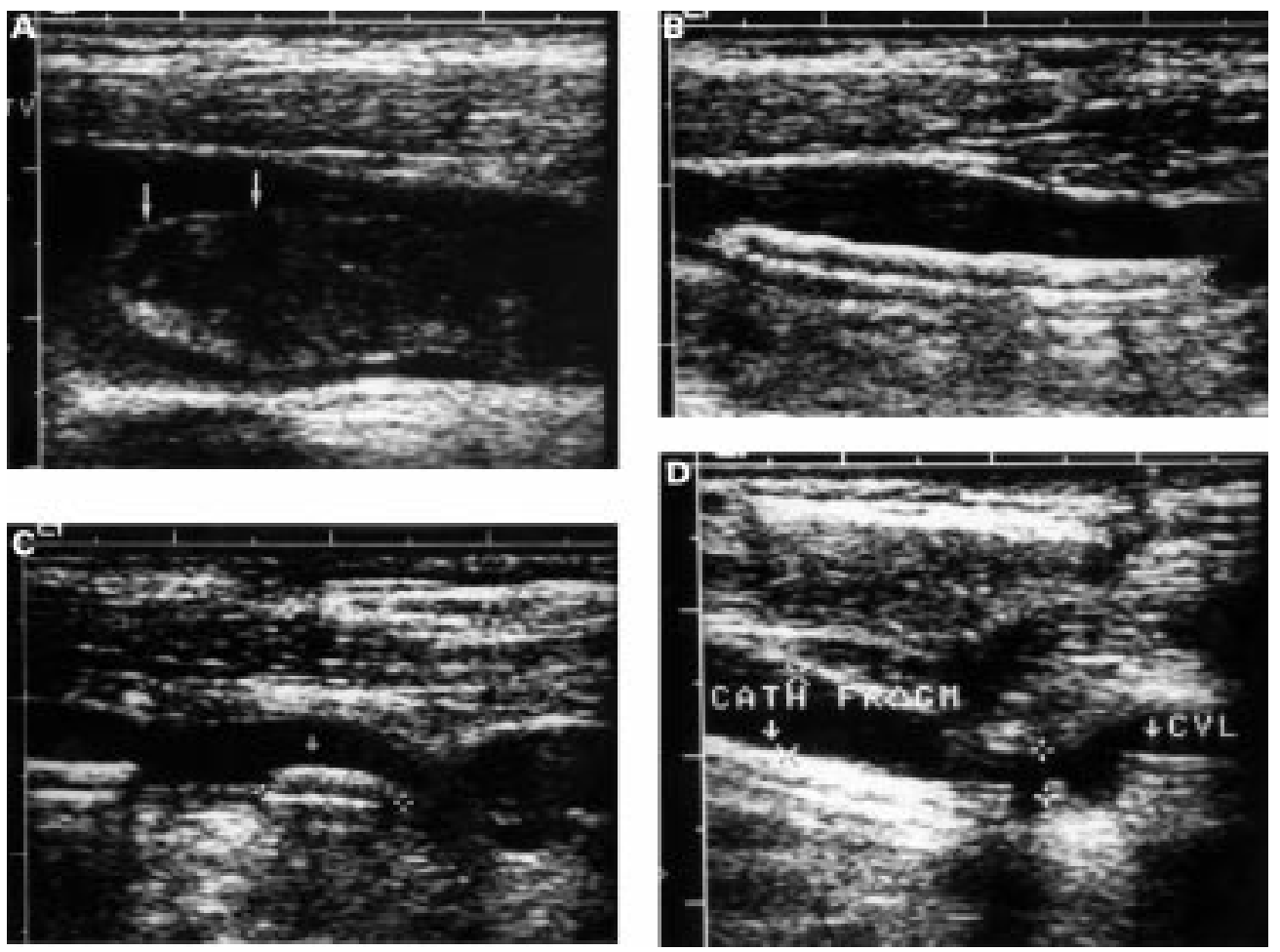

Figure 1 (A) Longitudinal sonogram of left internal jugular vein demonstrating echogenic thrombus (arrows) distending its lumen. (B) Longitudinal sonogram of right internal jugular vein demonstrating $3 \mathrm{~cm}$ long catheter fragment (between cursors) adherent to its posterior wall. (C) Longitudinal sonogram of right internal jugular vein, more central than (B), demonstrating the smallest catheter fragment (between cursors). (D) Longitudinal sonogram of right internal jugular vein demonstrating the central stenosis (between the two cursors in centre of image; $C V L=$ new central venous line).

fragment (fig 1). Despite thrombolytic therapy and catheter removal, this patient continued to have episodic facial swelling.

A second patient had two episodes of arm swelling due to thrombosis in the right internal jugular vein and right subclavian vein 1082 days after placement of the initial TIVAD and 497 days after replacement of the TIVAD. There were no episodes of pulmonary embolus identified in this series.

Five devices required repositioning of the catheter or porta. In three, the catheter was displaced outside the vein and required removal (table 2). The tip of one catheter was lying adjacent to the tricuspid valve and was repositioned. The position of one porta lay too close to the breast of a 16 year old girl, causing problems with both insertion of needles and pressure by bra straps. Repositioning of the porta led to a satisfactory result. Four devices were difficult to inject. All were managed successfully by ceasing use of the TIVAD, reinsertion of the needle, or by heparin saline flush. One porta had become mobile and required refixing to the chest wall without removal.

\section{INFECTIOUS COMPLICATIONS}

There were 18 infectious complications in 11 devices (table 1). This led to removal of the catheter in five of 57 devices (9\%). The median duration of catheter insertion prior to infection was 437 days (range 4-1049). The median duration of catheter functioning before removal due to an infectious complication was 697 days (range 6-1049).

There were 13 episodes of site infection, defined as signs of local skin infection over the infusion device without evidence of systemic infection. These were all treated successfully by ceasing use of the device, application of local wound care, and administration of systemic antibiotics via a peripheral intravenous line. The organisms cultured from the puncture sites were: coagulase negative Staphylococcus (2), Staphylococcus aureus (1), Acinetobacter (1), and Pseudomonas aeruginosa (1). These devices had been in place for a median duration of 422 days at the onset of infection (range 4-873).

Systemic infection occurred in five of the 57 devices $(9 \%)$, defined as clinical signs of sepsis with a positive culture from the portal site, with or without positive blood cultures. Two patients had Gram negative septicaemia. The organisms isolated from both blood and portal site were Pseudomonas maltophilia (1) and Flavibacterium (1). Both patients responded well to peripheral intravenous antibiotics and removal of the catheter. One child had Candida parapsilosis isolated from both peripheral blood and portal site. The child responded well to catheter removal and oral fluconazole. Two children developed signs of sepsis with a positive culture ( $S$ aureus and $P$ aeruginosa) from the portal site only. Both responded to broad spectrum antibiotics and removal of the catheter.

\section{DEVICE REMOVAL}

Twenty three of the 57 devices $(40 \%)$ were removed because of complications. Of these, six were replaced without further complications, nine were replaced with further complications, and eight were not replaced. Three devices were electively removed following 
successful lung transplantation and one device was electively removed because clinical improvement no longer necessitated frequent intravenous therapy. Fourteen children died from respiratory failure with the device in situ. Sixteen devices were in regular use and functioning well at the time of review.

\section{DOPPLER ULTRASONOGRAPHY}

Ten of the 16 patients (63\%) with functioning devices at the time of review underwent venous ultrasonography with Doppler to detect silent thrombosis. The median functional duration at the time of ultrasound was 902 days (range 195-3348). Ultrasonography findings were normal in eight patients. Two patients had evidence of early thrombus with a functional catheter duration of 1838 and 3348 days, respectively.

\section{Discussion}

The use of totally implantable venous access devices is an effective method of providing intravenous access for antibiotic therapy in patients with cystic fibrosis. These devices are not without risk, however. In this study complications occurred in $54 \%$ of devices, or one in every 1105 catheter days, and a major complication leading to the removal of the device was reported in $40 \%$ of devices, or one in every 2307 catheter days.

The percentage of complications per device in this study is higher than in two previous studies where complications occurred in $20 \%$ of devices over two years ${ }^{2}$ and $36 \%$ of devices over six years. ${ }^{4}$ The incidence of complications per catheter day is comparable with previous studies, however, ranging from one per 516 catheter days to one per 1482 catheter days. ${ }^{2-5}$ The longer follow up period of this study is one explanation for the greater complication rate identified.

Venous thromboses are a common complication of TIVADs. Catheter occlusion, presumably due to the presence of small thrombi within or at the tip of the catheter, and venous thrombosis led to device removal in 12 of the 57 devices $(21 \%)$, and symptomatic venous thrombosis occurred in five devices in four patients $(9 \%)$. While there are reports of the efficacy and safety of thrombolytic therapy in patients with TIVADs, ${ }^{78}$ none of our patients responded to this therapy and all required removal of the TIVAD. A range of thrombotic complications including pulmonary emboli, right atrial thrombus, chylothorax, sepsis, and death have all been reported ${ }^{45-14}$ but were not identified in this study. However, ultrasonography with Doppler detected early thromboses in two out of 10 asymptomatic patients.

David et $a l^{15}$ reported that central venous catheter related thrombotic complications were the most frequent cause of deep vein thrombosis in paediatric patients. Regular flushing of the device with heparin saline after use and monthly flushes when not in use, the standard method of maintaining a clear line, may not be sufficient in this group of patients. Sola et $a l^{4}$ recommend the prophylactic use of aspirin in all patients with cystic fibrosis without evi- dence of liver disease or coagulopathy, but there are few data currently available to support this recommendation. A randomised controlled trial of low dose warfarin in adults with cancer reduced central venous line related deep venous thrombosis diagnosed by venography from $38 \%$ to $10 \%$ without increased bleeding. ${ }^{16}$ However, the risk of haemoptysis is a particular concern in patients with cystic fibrosis.

The current gold standard for diagnosing central deep venous thrombosis is bilateral upper limb venography, ${ }^{10}$ an invasive procedure. Our study demonstrates the effectiveness of ultrasonography in diagnosing early thrombosis. Periodic sonographic monitoring to identify patients at risk and early treatment with thrombolytic therapy may reduce the risk of venous thrombosis in these patients.

The percentage of infectious complications ( $19 \%$ or one per 2948 catheter days) is comparable with previous studies which have reported a rate of infectious complications of $9-20 \%$ or one per 2006 to one per 5929 catheter days..$^{2-5}$ The high incidence of local infection and the predominant isolation of Gram positive cocci suggest direct inoculation or migration of the organism along the accessing needle as the primary mechanism of infection. Continuous staff education regarding aseptic technique with TIVADs has been reported to be the most effective method of preventing catheter related sepsis. ${ }^{17}$

The present catheter related rate of septicaemia of five in 57 devices (9\%) over nine years is comparable to reports of $6 \%$ over eight years, ${ }^{5}$ $9 \%$ over six years, ${ }^{4}$ and $9 \%$ over four years. ${ }^{3}$ All three patients in our study who had organisms isolated from both blood and portal sites were on treatment with oral steroids. Candida septicaemia was found in one of these patients. Candida species have frequently been reported as a cause of septicaemia leading to catheter removal in patients with cystic fibrosis and TIVAD. ${ }^{4-618}$ Bhargava et $a l^{19}$ reported fungal infections in $21 \%$ of 63 patients with cystic fibrosis at necropsy. All but one of these patients had central venous catheters in situ, and all four patients with disseminated fungal disease had indwelling central venous catheters. The risk factors for Candida septicaemia in patients with cystic fibrosis and a TIVAD include the combination of severe respiratory deficiency, an acute respiratory exacerbation, malnutrition, repeated and frequent broad spectrum antibiotic therapy, parenteral nutrition, and diabetes mellitus. ${ }^{20}$ The use of prophylactic antifungal treatment in patients with cystic fibrosis with a TIVAD has been suggested..$^{18} 19$

All devices in this review were inserted under general anaesthesia. This occurred safely, even in patients with poor pulmonary function. There were no pneumothoraces or intraoperative complications in this study, in comparison with a pneumothorax rate of $4 \%$ in a study where most procedures took place under local anaesthesia. ${ }^{3}$ However, an infectious complication on day 6 after insertion of a second device 
in this study may relate to surgical technique or postoperative wound care.

Correct positioning of the device is important. This complication can be reduced by carefully choosing the site to avoid interference with chest physiotherapy and clothing. In females TIVADs should be carefully sited away from the breast area. Internally, the site of the catheter tip should be confirmed radiologically to minimise the risk of interference of heart rhythm and to detect any residual catheter fragments after removal.

La Quaglia et $a l^{1}$ identified age less than seven years as a significant predictor of device related septicaemia. Ross et $a l^{12}$ reported that prematurity, total parenteral nutrition, continuous catheter use, but not duration of catheter placement increased the risk of thrombosis. In our study we failed to identify any differences in the group with and without complications. Specifically, there were no differences in age, sex, pulmonary function tests at the time of first insertion, number of courses of antibiotics per year, steroid therapy, duration of insertion, and death in the groups with and without complications. The complication rate was no different between the group who were taught to flush their own devices and the group where the device was flushed by trained staff. Duration of catheter insertion in this study did not predict complications leading to removal.

We conclude that the use of a TIVAD is an effective long term method of facilitating intravenous access in patients with cystic fibrosis who have limited peripheral venous access and require frequent intravenous therapy. There are, however, considerable risks associated with TIVADs and placement should be reserved for carefully selected patients. Careful preoperative preparation, meticulous surgical technique, staff and patient education regarding TIVAD aseptic technique may all reduce the number of complications. Periodic monitoring with ultrasonography and early thrombolytic therapy may help to reduce device removal for thrombotic complications. Duration of catheter placement is not a risk for complications and catheters can be left safely in situ until complications occur. Administration of a prophylactic anticoagulant should be considered and weighed against the risk of haemo- ptysis and bleeding. Prophylactic antibiotics and antifungal agents may have a role in patients at risk for catheter septicaemia but they need further evaluation in a prospective study.

1 Pattison J, Heaf DP. Totally implantable vascular access in treatment of cystic fibrosis. Lancet 1986; $\mathrm{i}: 799$.

2 Cassey J, Ford WDA, O'Brien L, et al. Totally implantable system for venous access in children with cystic fibrosis. Clin Pediatr 1988;27:91-5

3 Morris JB, Occhionero ME, Gauderer MWL, et al. Totally implantable vascular access devices in cystic fibrosis: a four year experience with fifty-eight patients. F Pediatr 1990;117: $82-5$

4 Sola JE, Stone MM, Wise B, et al. Atypical thrombotic and septic complications of totally implantable venous access devices in patients with cystic fibrosis. Pediatr Pulmonol 1992;14:239-42.

5 Yung B, Campbell IA, Elborn JS, et al. Totally implantable venous access devices in adult patients with cystic fibrosis. Respir Med 1996;90:353-6.

6 Stead RJ, Davidson TI, Duncan FR, et al. Use of a totally implantable system for venous access in cystic fibrosis.

7 Gray BH, Olin JW, Graor RA, et al. Safety and efficacy of thrombolytic therapy for superior vena cava syndrome. thrombolytic therapy

8 Peckham DG, Hill J, Manhire AR, et al. Resolution of superior vena cava obstruction following thrombolytic therapy in a patient with cystic fibrosis and a long-term indwelling catheter. Respir Med 1994;88:627-9.

9 Mollitt DL, Golladay ES. Complications of TPN catheterinduced vena caval thrombosis in children less than one year of age. F Pediatr Surg 1983;4:462-7.

10 Andrew M, Marzinotto V, Pencharz P, et al. A crosssectional study of catheter-related thrombosis in children receiving total parenteral nutrition at home. $\mathcal{F}$ Pediatr 1995; 126:358-63.

11 Korones DN, Buzzard CJ, Asselin BL, et al. Right atrial thrombi in children with cancer and indwelling catheters. $\mathcal{F}$ Pediatr 1996;128:841-6.

12 Ross P, Ehrenkranz R, Kleinman CS, et al. Thrombus associated with central venous catheters in infants and children. 7 Pediatr Surg 1989;24:253-6.

13 Monreal M, Raventos A, Lerma R, et al. Pulmonary embolism in patients with upper extremity DVT associated to venous central lines: a prospective study. Thromb Haemost 1994;72:548-50.

14 Raad II, Luna M, Khalil SA, et al. The relationship between the thrombotic and infectious complications of central venous catheters. FAMA 1994;271:1014-6.

15 David M, Andrew M. Venous thromboembolism complications in children: a critical review of the literature. $\mathcal{F}$ Pediatr 1993;123:337-46.

16 Bern MM, Lokich JJ, Wallach SR, et al. Very low doses of warfarin can prevent thrombosis in central venous catheters: a randomised prospective trial. Ann Intern Med 1990;112:423-8.

17 Daghistani D, Horn M, Rodriguez Z, et al. Prevention of indwelling central venous catheter sepsis. Med Pediatr Oncol 1996;26:405-8.

18 Fahy JV, Keoghan MT, Crummy EJ, et al. Bacteraemia and fungaemia in adults with cystic fibrosis. F Infect 1991;22: $241-5$.

19 Bhargava V, Tomashefski JF, Stern RC, et al. The pathology of fungal infection and colonisation in patients with cystic fibrosis. Hum Pathol 1989;20:977-86.

20 Horn CK, Conway SP. Candidaemia: risk factors in patients with cystic fibrosis who have totally implantable venous access systems. F Infect 1993;26:127-32.

21 La Quaglia MP, Lucas A, Thaler HT, et al. A prospective analysis of vascular access device-related infections in children. F Pediatr Surg 1992;27:840-2. 